\title{
Living Cells and Dynamic Molecules Observed with the Polarized Light Microscope: the Legacy of Shinya Inoué
}

\author{
TOMOMI TANI, MICHAEL SHRIBAK, AND RUDOLF OLDENBOURG* \\ Marine Biological Laboratory, Woods Hole, Massachusetts 02543
}

\begin{abstract}
In 1948, Shinya Inoué arrived in the United States for graduate studies at Princeton. A year later he came to Woods Hole, starting a long tradition of summer research at the Marine Biological Laboratory (MBL), which quickly became Inoué's scientific home. Primed by his Japanese mentor, Katsuma Dan, Inoué followed Dan's mantra to work with healthy, living cells, on a fundamental problem (mitosis), with a unique tool set that he refined for precise and quantitative observations (polarized light microscopy), and a fresh and brilliant mind that was unafraid of challenging current dogma. Building on this potent combination, Inoué contributed landmark observations and concepts in cell biology, including the notion that there are dynamic, fine structures inside living cells, in which molecular assemblies such as mitotic spindle fibers exist in delicate equilibrium with their molecular building blocks suspended in the cytoplasm. In the late 1970s and 1980s, Inoué and others at the MBL were instrumental in conceiving video microscopy, a groundbreaking technique which married light microscopy and electronic imaging, ushering in a revolution in how we know and what we know about living cells and the molecular mechanisms of life. Here, we recount some of Inoué's accomplishments and describe how his legacy has shaped current activities in polarized light imaging at the MBL.
\end{abstract}

Received 17 March 2016; accepted 4 August 2016.

To whom correspondence should be addressed. E-mail: rudolfo@mbl. edu

Abbreviations: CCD, charge-coupled device; DIC, differential interference contrast; "FluoPolScope," fluorescence polarizing microscope; LCPolScope, liquid crystal-polarizing microscope; OI-DIC, orientationindependent differential interference contrast; OPL, optical path length; polychromatic polscope, polychromatic polarized light microscope; SLCpolscope, single liquid crystal-polarization microscope.

\section{Introduction}

Before 1950, the primary tools of the cell biologist were the light microscope and the staining methods that highlighted specific structures in otherwise transparent cells. Although stains enhanced visibility, they interfered with the living process and even required fixing cells while trying to preserve their structures of interest. With the invention of the electron microscope in the 1930s (Ruska, 1980), and during its heyday in the 1950s and 1960s, much finer structural details could be discerned in cells that were fixed, stained, embedded in resin, and sliced for this new and captivating imaging tool. Results, however, depended on the fixing, embedding, and staining procedures used, a fact that electron and light microscopy seemed to have in common at the time.

Against this backdrop, Shinya Inoué and his mentor, Katsuma Dan, of Tokyo University, insisted on making observations on healthy, living cells, using the light microscope and non-invasive optical means to enhance contrast and highlight specific cell structures. They considered polarized light microscopy as a particularly promising tool, as it reveals order at a molecular scale that is usually hidden to the light microscope. By showing the alignment of molecular bonds and submicroscopic shapes, polarized light offered a way to bridge the resolution gap between light and electron microscopy, while observing living cells. In the early 1950s, in the MBL's Lillie Auditorium Inoué demonstrated the existence of parallel submicroscopic fibers in the mitotic spindle by showing movies of actively dividing cells that had been taken in polarized light. Until then, many researchers had questioned the reality of those fibers, as well as their roles in the anaphase separation of chromosomes and the division of the cytoplasm itself.

In this article, we discuss the role of Shinya Inoué in the reemergence of light microscopy in cell biology, with a 
focus on polarized light microscopy. We begin with a short history of polarized light microscopy in biology before Inoué, and end by describing new polarized light microscopy techniques that were inspired by Inoué's work and developed at the MBL. These techniques exploit the polarization of light to follow cellular events, from the building of the mitotic spindle to the formation of septin structures, one molecule at a time.

Our article is necessarily selective and cannot present the breadth and depth of Shinya Inoué's scientific accomplishments. For a more complete treatment, we refer the reader to Inoué's insightful articles and books, some of which tell his own story about microscopes, living cells, and dynamic molecules (Inoué, 2008a).

\section{Polarized Light Microscopy for Imaging Living Cells}

Polarization is a fundamental property of light that we cannot detect directly because the human eye is not sensitive to polarization (in contrast to many animal eyes.) When light passes through or reflects off materials, including biological cells and tissues, its polarization is generally altered. This change is measured by the polarizing microscope, and it can be used to identify molecular order and follow events that otherwise remain unseen in the light microscope.

Polarized light microscopy in biology started more than 100 years ago with the study of muscle contraction. The term, "striated muscle," was coined from observations with the polarized light microscope that revealed birefringent (anisotropic) A-bands and isotropic I-bands in muscle tissue. The I-band is not strictly isotropic, but is less birefringent than the A-band, which is now known as the domain containing the thick filaments composed of myosin, including a region in which the thin actin filaments of the I-band overlap the myosin filaments. Already in the late $19^{\text {th }}$ century, in a visionary conjecture Georg Engelmann attributed the origin of contractility of striated muscle to the A-band (Engelmann, 1875, reviewed by A. F. Huxley in an essay edited by A. L. Hodgkin (Hodgkin et al., 1977)). Through the examination of many other contractile structures in various organisms, such as the body wall of Hydra, the contractile stalk of vorticellids and other ciliates, contractile protoplasm, and embryonic muscle cells, Engelmann concluded that wherever and in whatever form it may occur, contractility was linked to the presence of birefringence.

In the 1920s, Wilhelm J. Schmidt began to systematically observe animal cells and tissues with the polarized light microscope. He found that many structures in living tissue were birefringent, including muscle, connective tissue, bone, and even the mitotic spindle in dividing cells (Fig. 1). Schmidt published his findings in two important monographs (Schmidt, 1924, 1937) that, to this day, represent the
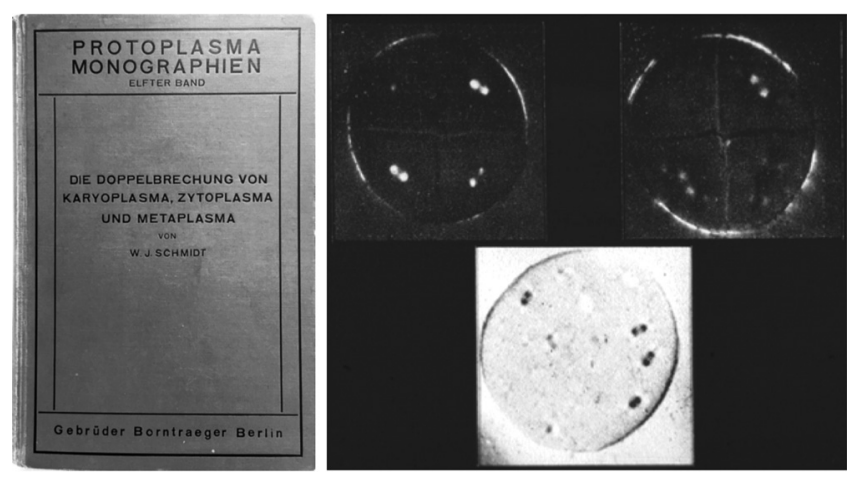

Figure 1. Observations of birefringence of the mitotic spindle (right side) were reported by W. J. Schmidt in the second of his monographs on the birefringence of animal cells and tissues (Schmidt, 1937). (Reprinted from panels a, b, and c, p. 89, of Schmidt, W. J. (1937), Die Doppelbrechung von Karyoplasma, Zytoplasma und Metaplasma, with permission from Schweizerbart Science Publishers, Stuttgart; www.schweizerbart.de.)

most comprehensive survey and analysis of structural features of biological materials observed in polarized light.

Inspired by the findings of W. J. Schmidt and prompted by Katsuma Dan, Shinya Inoué started, in the 1940s, to build his own polarizing microscope. He went on to reproduce Schmidt's observations of the birefringence of the living spindle. There were two challenges for young Shinya Inoue to overcome in detecting the weak birefringence of mitotic spindles. (See Inoué's personal reminiscence of this period (Inoué, 2008b).) The first challenge was in finding a way to lower the polarization distortions caused by every optical component in the microscope. After crossing the analyzer and polarizer in the microscope, he found that the back focal plane of the objective lens, between two crossed polarizers (extinction condition), was not as uniformly dark as expected. He discovered that polarization distortions caused by optical components in the microscope light path (curved lens surfaces, strained glass, and mirrors) could brighten the back aperture image of the objective lens. This background light significantly lowered the signal-to-noise ratio for detecting the weak intensity that identified spindle structures. To overcome this problem, he turned the lenses along the optical axis until the effects of undesirable birefringence in the strained condenser and objective lenses canceled each other out.

Once Inoué achieved a dark back aperture between two crossed polarizers, he encountered the next challenge: while the image of the spindle in an actively dividing cell was now visible to the naked eye, the contrast was insufficient to record on film the transient spindle birefringence. In fact, turning the lenses to gain maximum extinction had effectively eliminated an optical element that, under the right circumstances, can help enhance the contrast of birefringent objects.

That element was a uniformly birefringent plate, called a compensator, which had to be placed between the crossed 

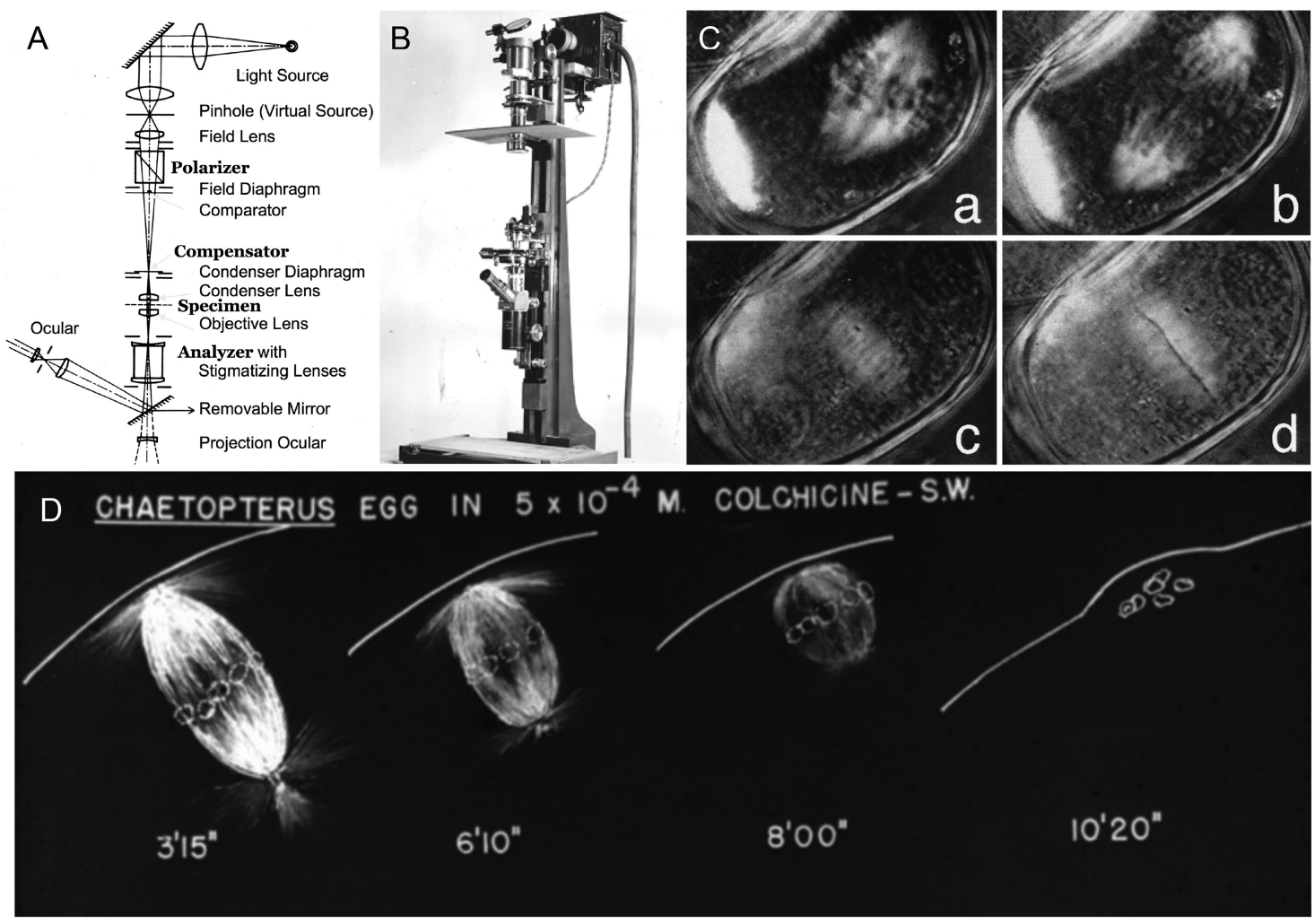

Figure 2. Polarized light microscope. (A) Schematic and (B) photograph of the Shinya-Scope Nr. 2 (Inoué, 1951), which may well have been the first inverted microscope used in biology. (C) Mitosis and cell plate formation in a centrifuged pollen mother cell of the Easter lily Lilium longiflorum, viewed by polarization microscopy and recorded on 16-mm celluloid film in 1952 (Inoué, 1981a). (Reproduced from figure 6 of S. Inoué (1981), Journal of Cell Biology 91: 131s-147s.) (D) Schematic of the shortening and loss of birefringence of a metaphase-arrested, Chaetopterus spindle, exposed to colchicine in seawater. Below each frame is the time in minutes and seconds after the application of $5 \times 10^{-4} \mathrm{~mol}^{-1}$ colchicine (Inoué, 1952a). (Reprinted with permission of Elsevier from Inoué (1952), Exp. Cell Res. Suppl. 2: 305-308. Permission conveyed through Copyright Clearance Center, Inc.)

polarizers, either before or after the specimen. Without a compensator, a specimen with birefringence as low as that of the mitotic spindle in a living cell would not generate enough image brightness and contrast to successfully expose a film. However, when a weakly birefringent compensator was added between the crossed polarizers, the microscope field of view became a dark gray instead of nearly black. More important, the appearance of the spindle depended on its orientation with respect to the birefringence axis (slow axis) of the compensator. When the slow axis was more parallel to the spindle axis, its fibers appeared bright and at a higher contrast to the rest of the field of view. Inoué built a microscope that fulfilled all of these conditions, making it possible to record historic time-lapse movies of mitosis in actively dividing cells (Fig. 2) (Inoué, 1953).

In addition, Inoué noted the reversal of contrast when orienting the spindle axis more perpendicular to the slow axis of the compensator, an observation that unambiguously assigned a positive birefringence to the material that formed the spindle. The positive birefringence was compatible with the notion that the spindle is composed of submicroscopic, parallel fibers spanning between the poles and the chromosomes. This observation finally settled the dispute about the reality of spindle fibers and their role in segregating chromosomes in the living cell, a hotly debated issue at the time.

Inoué's polarized light movies unequivocally demonstrated the existence of parallel fibers-now known as microtubules-inside the living, actively dividing cell. The demonstration rested on the birefringence of the parallel fibers and their effect on the polarization of light, while the individual fibers themselves remained hidden to the light microscope. The definitive proof that spindle birefringence is caused by parallel arrays of microtubules came decades later, after appropriate fixation methods were developed that preserved the labile microtubule structure (Olmsted and Borisy, 1973). These advances by others enabled Inoué and colleagues to observe the same spindle first by polarized light and then, after fixation, staining, and sectioning, by electron microscopy, to identify the number of microtubules contributing to the birefringence of the spindle (Sato et al., 1975). 

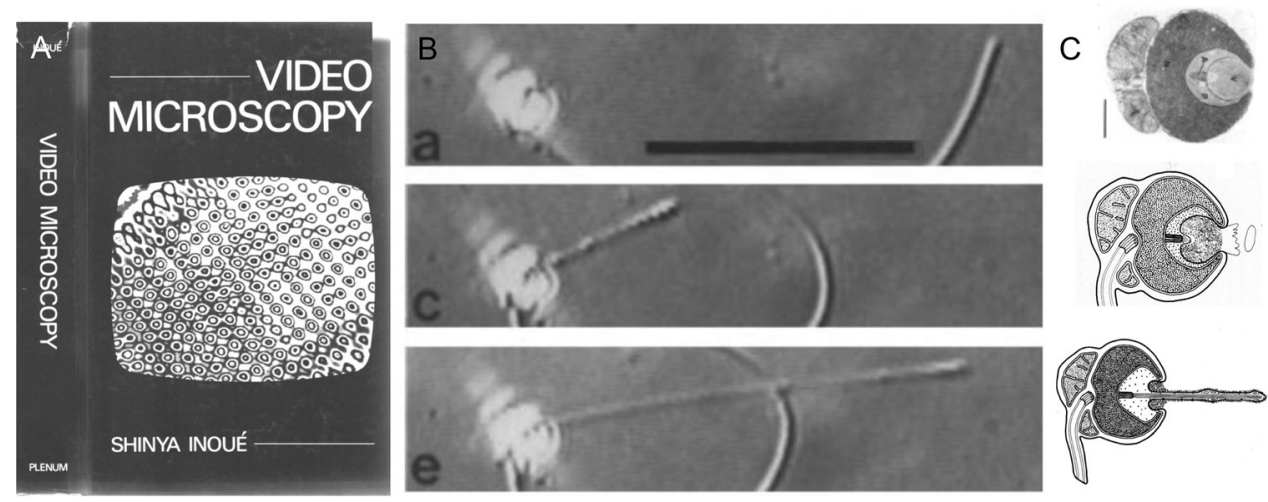

Figure 3. Video microscopy of the acrosomal reaction (growth of 65-nm diameter acrosomal process by actin polymerization) in Thyone sperm. (A) Book cover of the first comprehensive treatise on video microscopy, merging the fields of microscopy, electronic imaging, and digital image processing and analysis (Inoué, 1986). (B) Three stages, 1.5 seconds apart, in acrosomal reaction and elongation of the acrosomal process, which is less than 100-nm thick. Scale bar $=20 \mu \mathrm{m}$. (C) Electron micrograph (top) and schematics of structural changes in Thyone sperm undergoing the acrosomal reaction (Inoué and Tilney, 1982; Tilney and Inoué, 1982). (Modified from figure 6 of S. Inoué and L. G. Tilney (1982), Journal of Cell Biology 93: 812-819.)

In subsequent studies, Inoué and collaborators established the reversible assembly and disassembly of spindle fibers and fibrils in living cells when exposed to cold temperatures (Inoué, 1964), antimitotic drugs (Inoué, 1952a), hydrostatic pressure (Salmon, 1975), and exchange of regular water with heavy water (Inoué and Sato, 1967). In each of these studies, polarized light microscopy was the key to observing the submicroscopic, fine structure of the spindle. Its dynamic architecture could be followed in real time, at high temporal and spatial resolution, and with no staining or labeling required.

The results with colchicine were especially intriguing (Fig. 2D). When metaphase-arrested oocytes of Chaetopterus were exposed to colchicine, the spindle birefringence gradually disappeared as the fibrils depolymerized (the kinetochore fibers were the longest to persist), but the depolymerizing filaments actually led the chromosomes and inner spindle pole to the cell surface, where the outer meiotic spindle pole was attached. Thus, the colchicine experiments suggested that depolymerizing filaments could generate forces adequate to pull the chromosomes and the spindle poles toward each other. This interpretation ran against the dogma of the time, yet was visionary and proven correct many decades later (Inoué and Salmon, 1995).

Inoué and collaborators further improved the sensitivity and resolution of the polarizing microscope by adding polarization rectifiers, which counteracted the systematic polarization distortions of high-resolution objective and condenser lenses (Inoué, 1952b; Inoué and Hyde, 1957). These improvements made possible many new observations, including another landmark study in the 1960s, in which Inoué revealed the packing of DNA in living sperm heads by interpreting his polarized light observations in terms of the structure of DNA, a feat that linked light microscopy with the new discipline of molecular biology (Inoué and Sato, 1966).

\section{Video Microscopy}

Around 1980, Shinya Inoué and his colleague, Robert D. Allen, introduced an important advance in light microscopy: the addition of a high-quality video camera to the microscope. The initial intent was simply to display the microscope field of view to a larger group of viewers, attendees of either of the two MBL microscopy courses, one organized by Allen and the other, by Inoué. The video equipment turned out to have unexpected capabilities, and established the video camera as a permanent fixture on the researchgrade microscope. For example, by matching the camera's resolution to the optical resolution of the microscope and manipulating the electronic controls in the video circuitry, image contrast could be boosted and the finest details near the resolution limit of the light microscope, usually hidden to the human eye, made clearly visible. With the aid of image computations, images could be refined by enhancing contrast, subtracting away unwanted background, and enhancing image features at selected spatial frequencies so as to detect images otherwise far too faint to be seen. The practical applications of video-enhanced microscopy were first explored with the differential interference contrast (DIC) microscope by Allen and collaborators (Allen et al., 1981), and the polarizing microscope by Inoué (Inoué, 1981b). The video enhancement method, combined with high numerical aperture (NA) objective lenses, increased the sensitivity of both label-free imaging methods for detecting submicroscopic objects, such as organelles, filaments, and membrane tubules, in the living cell and in real time (Fig. 3) (Inoué, 1986). The combination of video 
recording with digital image processing and analysis further enhanced the utility of the light microscope as a quantitative tool for studying living cells and tissues in their physiological environments. Quickly, the new technique was adopted in most every mode of light microscopy used in biology today.

In an early application of video-enhanced DIC, Inoué and Lewis Tilney (1982) observed the acrosomal reaction of the sea cucumber sperm (Fig. 3B). Once the high-calcium seawater reached the sperm, the acrosome burst, followed by the rapid growth of a slender acrosomal process over the next several seconds. Initially, the process elongated at a speed of nearly $10 \mu \mathrm{m} \mathrm{s}^{-1}$ until gradually slowing down, reaching a final length of $50-75 \mu \mathrm{m}$ in 7 to $8 \mathrm{~s}$. Videoenhanced DIC clearly visualized the growing acrosomal process, which was only $65 \mathrm{~nm}$ in diameter, about one fourth of the resolution limit of the light microscope, and could do so at 30 frames per s. The curve plotting the elongation process fits a diffusion-limited event. This implies that unpolymerized actin molecules diffuse within the thin, growing membrane tube all the way to the tip of the acrosomal process, where they are polymerized onto growing ends of the actin filaments, which support the growth of the process (Inoué and Tilney, 1982).

\section{Enhancing Polarized Light Microscopy Using Liquid Crystal Devices and Digital Image Processing}

Electronic image capture and digital image processing brought new opportunities with which to exploit the quantitative nature of polarized light microscopy. For the first time, instantaneous measurements of intensities in every resolved image point were possible; images could be converted into digital format, stored, and immediately processed. In addition, liquid crystal (LC) devices suitable for imaging and instantly manipulating the polarization of light became available. Recognizing the revolution in light microscopy, Rudolf Oldenbourg joined Shinya Inoué at the MBL in 1989. With Inoué's support, he developed the LC-polarizing microscope (LC-PolScope) (licensed to PerkinElmer, Inc., Waltham, MA), which significantly advanced the utility of the polarized light microscope in biology and in many other areas of application (Oldenbourg, 1996).

The optical design of the LC-PolScope builds on the traditional polarizing microscope, introducing two essential modifications: the specimen is illuminated with nearly circularly polarized light, and a universal compensator replaces the traditional compensator (Fig. 4A) (Oldenbourg and Mei, 1995; Shribak and Oldenbourg, 2003). The universal compensator is built from two LC-based, variable retarders and a linear polarizer. Each LC plate has a uniform retardance that depends on the voltage applied to the device. An electronic controller connected to a computer supplies the voltages. The polarization analyzer for circularly polarized light, placed behind the objective lens, completes the polarization optical train. The specimen image, projected by the objective lens, is recorded by a charge-coupled device (CCD) or similar camera with a linear response to light intensity. Images are digitized and downloaded to the computer, with software synchronizing the image acquisition process with the LC settings and implementing imageprocessing algorithms. Several related image-acquisition and processing algorithms can be used to compute images that represent the retardance and slow-axis orientation in each resolved image point (Shribak and Oldenbourg, 2003). Figure 4A illustrates the 5-frame algorithm, which is preferred for imaging structures with low retardance. Later, Michael Shribak proposed the single LC-polarization microscope (SLC-polscope), which employs an alternative universal compensator (also known as a complete polarization state generator) that is built from a single LC-based variable retarder (the LC-PolScope has two LC retarders), a rotatable linear polarizer, and a quarter wave film (Shribak, 2011). The SLC-polscope uses a new, 3-frame algorithm.

Minor changes to the hardware and software of the LCPolScope also enable measurements of diattenuation or dichroism in a sample. These changes and the physical meaning of retardance, slow axis, diattenuation, and dichroism are discussed in the context of the LC-PolScope by Shalin B. Mehta et al. (2013). As first proposed by Inoué, polarization images created by high-NA objective lenses can be improved by incorporating polarization rectifiers into the illumination and/or imaging light paths (Inoué and Hyde, 1957; Shribak et al., 2002).

In addition to making the measurements quantitative, Oldenbourg followed Inoué's example by aiming for observations that could be interpreted in terms of cellular events at the molecular level. Important steps in that direction included measurements of the birefringence of single biopolymers, such as microtubules (Oldenbourg et al., 1998) and actin filaments (Katoh et al., 1996). Building on these results, James LaFountain and Oldenbourg quantified microtubule and chromosome dynamics in the meiotic spindle of crane fly spermatocytes (Fig. 4B, C), including the monitoring of microtubule numbers in kinetochore fibers in maloriented bivalents (LaFountain and Oldenbourg, 2004). They combined laser microsurgery with LC-PolScope observations to uncover the functional states of kinetochores and the maturation of kinetochore fibers during stages of cell division (LaFountain et al., 2011; LaFountain and Oldenbourg, 2014). Through their interdisciplinary collaboration with David Keefe, MD, the LC-PolScope also became a valuable tool in vitro fertilization clinics and stem cell laboratories around the world (Liu et al., 2000; Byrne et al., 2007). 

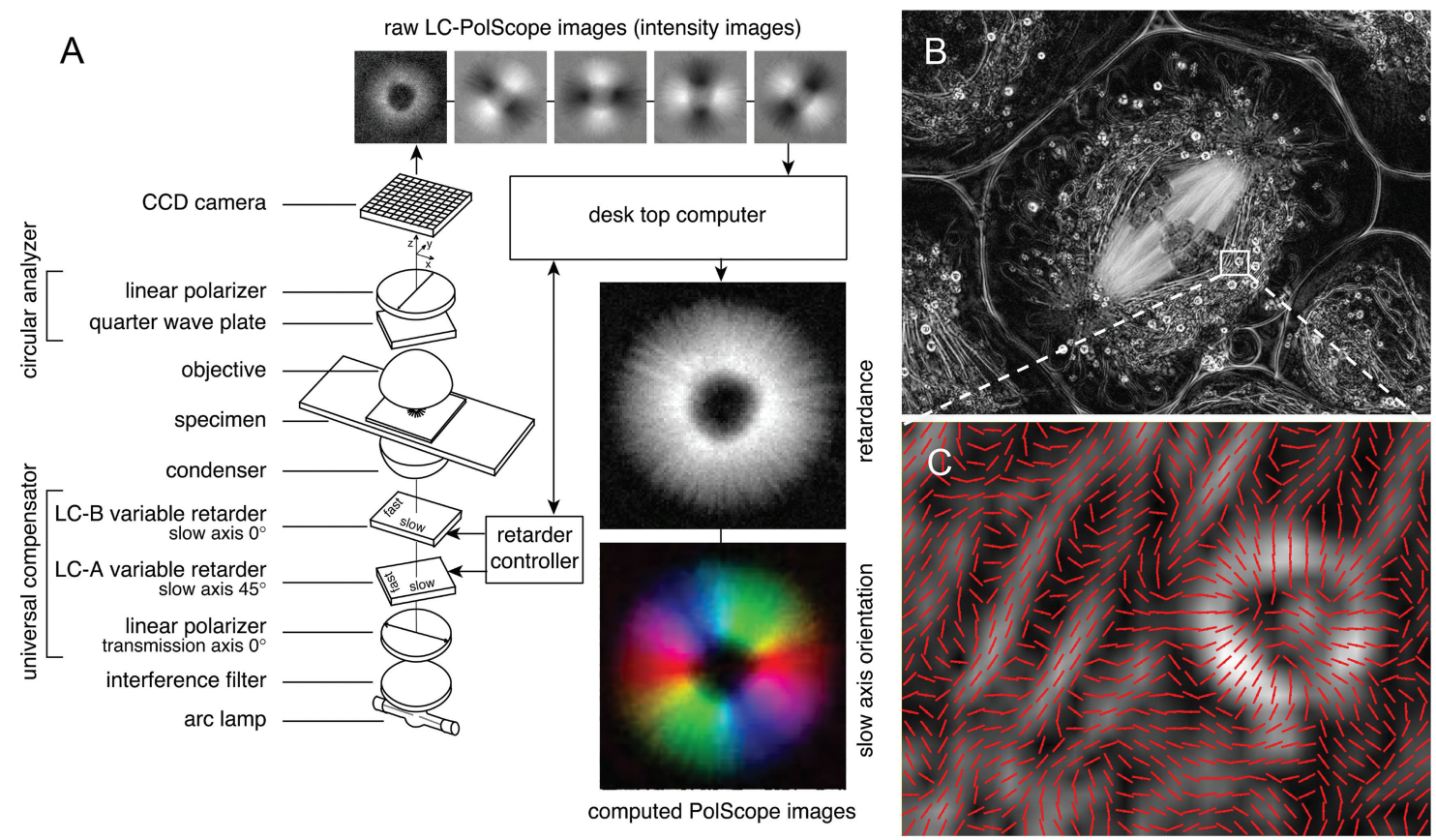

Figure 4. The liquid crystal-based polarizing microscope (LC-PolScope). (A) Schematic of the optical design (left), which builds on the traditional polarized light microscope, with the conventional compensator replaced by two liquid crystal (LC)-variable retarders, the LC-A and LC-B. The combination of microscope optics, electro-optic components, electronic imaging, and digital image processing was used to generate computed PolScope images of an aster, consisting of microtubule fibers radiating from a centrosome. (B) LC-PolScope image of a live, primary spermatocyte from the crane fly Nephrotoma suturalis. Brightness is directly proportional to the birefringence of the specimen, independent of the orientation of the birefringent structure. The flattened cell reveals with clarity the birefringence of spindle microtubules extending from the chromosomes to the spindle poles. The birefringence of other cell organelles, such as elongated mitochondria, surrounding the spindle like a mantle, and small spherical lipid droplets, are also evident against the dark background of the cytoplasm. The pole-to-pole distance of the spindle is approximately $25 \mu \mathrm{m}$. (C) Magnified cytoplasmic region of (B), with overlaying red lines indicating the slow-axis direction for each pixel. The lipid droplet at the right (white circular object) has a highly birefringent shell with slow axes perpendicular to the droplet's surface, while mitochondria have slow axes that are parallel to the mitochondria's long axes.

\section{The Polychromatic Polscope}

Inspired by Shinya Inoué, Michael Shribak devised a new technology, the polychromatic polscope, for direct viewing of a combination of retardance and slow-axis orientations in a specimen (Shribak, 2015).

The optical design of the polychromatic polscope is based on a standard polarized light microscope, which is equipped with a special polarization state generator and analyzer. The specimen is illuminated with white light, whose spectral components have different polarizations produced by the special polarization state generator. As the beam passes through the specimen, its birefringent structures modify the beam polarization in such a way that, after the special polarization analyzer, non-birefringent specimen parts appear black and birefringent structures, colored. The hue of the structure indicates its slow-axis orientation, and the brightness of the structure is proportional to its retardance, as illustrated by the rotifer image in Figure 5 .
The traditional polarizing microscope displays objects in Newton interference colors if their retardance is within the range of $400-2000 \mathrm{~nm}$. The hue is determined by the retardance value, as illustrated by the Michel-Levy chart (Oldenbourg and Shribak, 2010). This phenomenon is widely used for analyzing birefringent samples in mineralogy. However, many biological structures have retardance values that are less than $100 \mathrm{~nm}$. Therefore, cells and tissues viewed with a regular polarization microscope appear gray, with contrast disappearing at certain orientations.

Unlike the traditional polarizing microscope, the polychromatic polscope has interference colors that indicate the orientation of birefringent structures and do not depend on the retardance induced by the structures, as long as their retardance values are less than about $400 \mathrm{~nm}$. Such low-retardant structures appear gray in a traditional polarizing microscope, while in the polychromatic polscope they appear colored, with the hue 


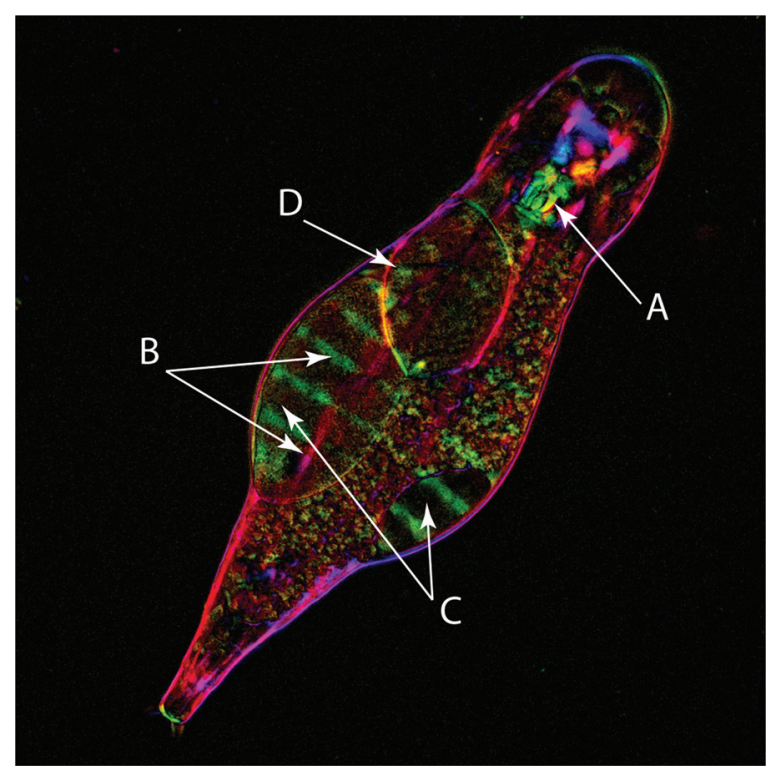

Figure 5. Polarization polychromatic image of bdelloid rotifer Adineta vaga, a microscopic freshwater invertebrate. Birefringent structures appear colored, with hue related to the slow-axis orientation. The image was recorded by Michael Shribak and Irina Arkhipova (MBL, Woods Hole, MA), using a color camera and the polychromatic polscope. This image of an adult female emphasizes characteristic structures such as the pharynx with a mastax consisting of hard jaws (trophi) (A); a set of circular and longitudinal muscles (B); bilateral ovaries with oocyte nuclei (C); and the parthenogenetically developing egg (D). The picture width is $280 \mu \mathrm{m}$, the acquisition time was $5 \mathrm{msec}$. (Modified from figure 3 in M. Shribak (2015), Scientific Reports 5: 17340.)

reporting the slow-axis orientation. With the polychromatic polscope, Shribak proposed, for the first time, the use of vector interference in polarized light imaging, in which the fullcolor spectrum appears even in specimens with low retardance of only a few nanometers, and images are recorded with a single snapshot. Previously colorless polarized light images of organelles, cells, and tissues become vividly colored, with their hue specifying the slow-axis orientation of molecular assemblies in living organisms. This approach can open up new possibilities for detecting structural signatures and dynamics of molecular assemblies in unlabeled specimens with weak birefringent structures, potentially aiding in the diagnosis of various diseases that relate to abnormal formation of molecular assemblies in cells affected by Alzheimer's, Huntington's, Parkinson's, and prion diseases, as well as sickle cell anemia and malaria (Shribak, 2015). Other applications will be found in the imaging of low-birefringent crystals and in creating new methods for controlling the colors of a light beam.

In addition, the polychromatic light polarization technique allows one to see a colored polarization image directly and to capture the picture instantly in real time. Therefore, it enables the highest temporal resolution, producing sharp images of fast-moving, low-birefringent struc- tures, and it makes visible rapid processes that are accompanied by changes in birefringence. In combination with a pulsed light source, the new microscope should make it possible to visualize nerve activity, shock wave propagation, and other fast-moving events.

We successfully applied the polychromatic polarization technique for imaging various types of fast-moving bdelloid rotifers. Bdelloid rotifers are microscopic freshwater invertebrates best known for their capacity to undergo frequent cycles of desiccation and rehydration at any life stage; for their long-term asexuality, which is manifested in the absence of males and meiosis; and for their ability to capture foreign genetic material at levels unprecedented in metazoans. A live image of the bdelloid rotifer Adineta vaga, as viewed with the polychromatic polarized light microscope under $20 \times$ magnification, is shown in Figure 5 .

\section{Orientation-Independent Differential Interference Contrast Microscopy Using Liquid Crystal Devices and Digital Image Processing}

Working with Shinya Inoué, Michael Shribak also devised the quantitative orientation-independent differential interference contrast (OI-DIC) microscope (Shribak and Inoué, 2006). The conventional DIC microscope shows the two-dimensional distribution of the optical phase gradient encountered along the shear direction between two interfering beams. Therefore, the contrast of DIC images varies with the orientation of the phase gradient with respect to the shear direction, giving DIC images their characteristic relief appearance. The image contrast also depends on the initial phase difference (bias) between the interfering beams. To overcome the limitations of traditional DIC systems, Shribak has developed a quantitative OI-DIC microscope, which allows the bias to be modulated and shear directions to be switched rapidly without mechanically rotating the specimen or the prisms (Fig. 6A) (Shribak, 2013). A set of raw DIC images with orthogonal shear directions and different biases is captured within a second. Specialized software first computes a phase gradient vector map and then a quantitative phase image (Fig. 6B).

The new OI-DIC technique can be used with any high-NA objective lens at full aperture, and it provides an optical path length (OPL) or phase shift map at the highest resolution attainable with the imaging optics. Unlike other phase-mapping techniques, the OI-DIC does not require phase unwrapping. It can be combined with the LC-PolScope to yield two complementary images: one showing dry mass distribution ( refractive index) and the other showing distribution of birefringence $(\sim$ structural or internal anisotropy). For example, the OI-DIC picture displays the chromosomes, while the LC-PolScope image shows the spindle fibers (Shribak et al., 2008). Combining fluorescence microscopy with OI-DIC imaging provides the molecular 

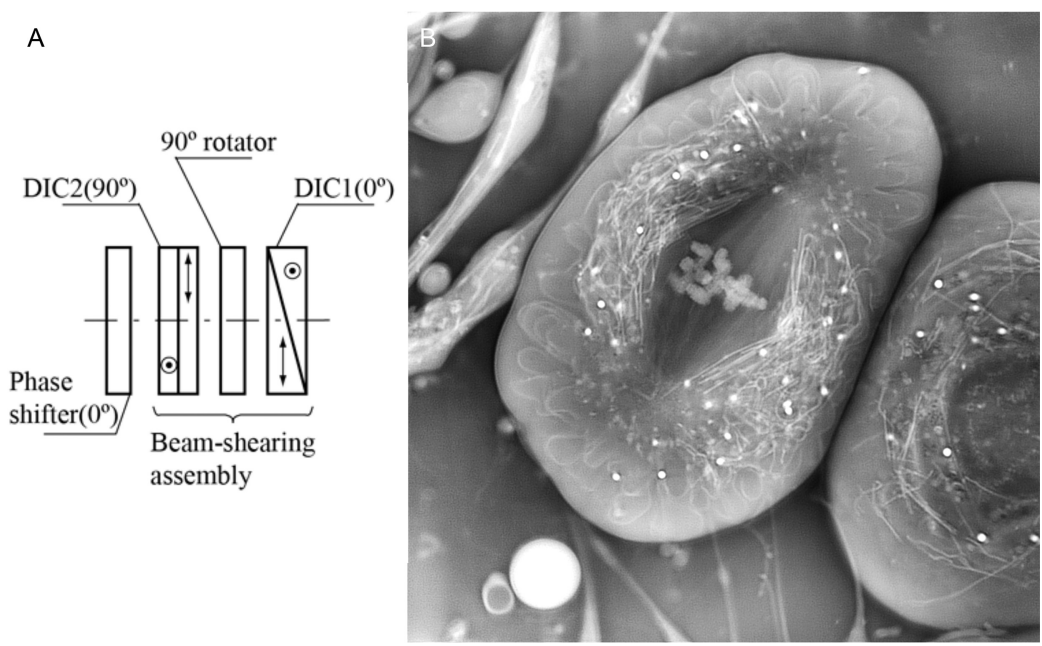

Figure 6. Orientation-independent differential interference contrast (OI-DIC) microscopy. (A) Schematic of the OI-DIC beam-shearing assembly, which consists of two standard DIC prisms, between which is a liquid crystal $90^{\circ}$ polarization rotator. The shear plane of the first prism (DIC1) is oriented at $0^{\circ}$, and the shear plane of the second prism (DIC2) is oriented at $90^{\circ}$. Another liquid crystal (LC) cell works as a phase shifter, which modulates the bias; its principal plane is oriented at $0^{\circ}$. We employed a twisted-nematic LC cell as a $90^{\circ}$ rotator and an untwisted nematic cell as phase shifter. (B) The brightness of the OI-DIC phase image of the crane fly spermatocyte (full metaphase of meiosis-I) is linearly proportional to the optical path length. The three autosomal, bivalent chromosomes are pulled apart at the spindle equator, along with one of the X-Y sex univalents located at the right. The distribution of tubular mitochondria surrounding the spindle and granual chromosome structure is clearly visible. The experiment was done by M. Shribak and J. R. LaFountain, Jr. (University at Buffalo, Buffalo, NY) (Shribak et al., 2008). David Biggs, of KB Imaging Solutions LLC, Loomis, CA, computed the phase image, using iterative deconvolution algorithms. Raw images were recorded on an Olympus microscope BX61, equipped with a UPlanFl 100 $\times / 1.30$ oil immersion objective lens and 546/30 nm interference filter. Image acquisition and processing took about 1 second each. Image size is 68 $\mu \mathrm{m} \times 68 \mu \mathrm{m}$. (Modified from figure 2.8, Chapter 2, pp. 19-42, M. Shribak (2013), Biomedical Optical Phase Microscopy and Nanoscopy, $1^{\text {st }}$ ed. Academic Press, New York.)

specificity of fluorescence and quantification of OI-DIC. The type of organelles in a live specimen could be scored using fluorescence markers, and subsequent organelle development followed during a long time series using OI-DIC, with minimal phototoxicity.

A standard research-grade light microscope equipped with OI-DIC and a $100 \times / 1.3$ NA objective lens, which was not specially selected for minimum wavefront and polarization aberrations, provides lateral resolution of about $250 \mathrm{~nm}$ and a discrimination depth of approximately $100 \mathrm{~nm}$ at a wavelength of $546 \mathrm{~nm}$. To the best of our knowledge, other currently available interference and phase microscopy techniques cannot achieve these parameters. The new OI-DIC technology is the next step in the development of DIC microscopy. It can replace standard DIC prisms in existing commercial microscope systems without modification. This feature will allow biological researchers who already have microscopy setups to expand the performance of their systems.

\section{Dissection of Protein Assembly in Living Cells Using Polarized Fluorescence Microscopy}

Birefringence is related to structural features such as the alignment of bonds and submicroscopic shapes, but it does not reveal the chemical nature of the molecules that build the anisotropic structures. Fluorescence labeling, on the other hand, is used to identify chemical building blocks, but usually does not report their submicroscopic alignment within the higher-order structure. Polarized fluorescence can combine both virtues, structural and molecular specificity. Shinya Inoué recognized this capability when he and his MBL colleague, Osamu Shimomura-who shared the Nobel Prize in Chemistry in 2008 with Martin Chalfie and Roger Tsien for the discovery of the green fluorescent protein (GFP) - that GFP crystals emit highly polarized fluorescence (Inoué et al., 2002), hinting at the polarized fluorescence emitted by individual GFP molecules. Inspired by this discovery, Alina Vrabioiu and Tim Mitchison designed an orientationally constrained GFP-septin construct, using it to determine septin filament organization and dynamics in living yeast (Vrabioiu and Mitchison, 2006, 2007).

Motivated by these breakthroughs, Oldenbourg and colleagues developed the liquid-crystal (LC)-based fluorescence polarizing microscope (fluorescence LC-PolScope), which sequentially excites fluorophores with linearly polarized light of four orientations: $0^{\circ}, 45^{\circ}, 90^{\circ}$, and $135^{\circ}$ (DeMay 
et al., 2011b; McQuilken et al., 2015). The use of four linear polarizations, rotated in 45-degree steps, enables measurement of polarized fluorescence that is oriented at any angle in the image plane. This was an improvement over the earlier approach by Daniel Axelrod (Alexrod, 1989), which used only two orientations parallel or perpendicular to a previously identified symmetry axis. The fluorescence LCPolScope was used to analyze the 3D architectural rearrangement of constrained, GFP-tagged septin assemblies during cytokinesis of budding yeast and the filamentous fungus Ashbya (DeMay et al., 2011a).

Despite the advancements made with the fluorescence LC-PolScope, the time needed to collect four sequential images limited its use for fast-moving or rearranging structures due to possible motion artifacts. To avoid spurious results, objects must not move faster than the resolution limit of the imaging optics over the time it takes to acquire all four images. This situation precludes observation of objects that move at roughly $1 \mu \mathrm{m} / \mathrm{s}$ or faster, including single fluorophores.

To overcome this limitation, Tomomi Tani and colleagues have developed an instantaneous fluorescence polarizing microscope (which they refer to as the instantaneous "FluoPolScope") (Mehta et al., 2016), which combines isotropic total internal reflection excitation and instantaneous sorting of emitted fluorescence along four polarization orientations, using a custom image splitter (Fig. 7A). This device is optimized for live-cell imaging with a high-sensitivity, low-noise camera and image analysis that can identify and even track single molecules (Fig. 7B). Tani et al.'s method promises to answer important questionsraised by studies using high-resolution X-ray crystallography, nuclear magnetic resonance (NMR) imaging, and electron microscopy-about dynamics of ordered assemblies in living cells and systems reconstituted in vitro.

\section{Polarized Light Microscopy in the 21st Century}

For more than a century, polarized light microcopy has provided submicroscopic structural signatures of label-free molecular assemblies in living organisms and tissues. It has revealed submicroscopic alignments that are usually invisible to the light microscope (Valentin, 1861; Engelmann, 1875). In the 1930s, W. J. Schmidt extended these studies to single animal cells and their components in the nucleus and cytoplasm (Schmidt, 1937, 1939). The 3D architecture of membrane lipid bilayers, chromosomes, and cytoskeletons is regulated by the interaction between their component molecules. Thus, monitoring their orientation and mutual alignment provides important clues for understanding the regulatory mechanisms that govern the architectural dynamics of these assemblies, which, in turn, generate the large variety of cell shapes and functions in time and space. The ordered alignment of molecules, which can be detected as

\section{A 4-way polarization splitting system}

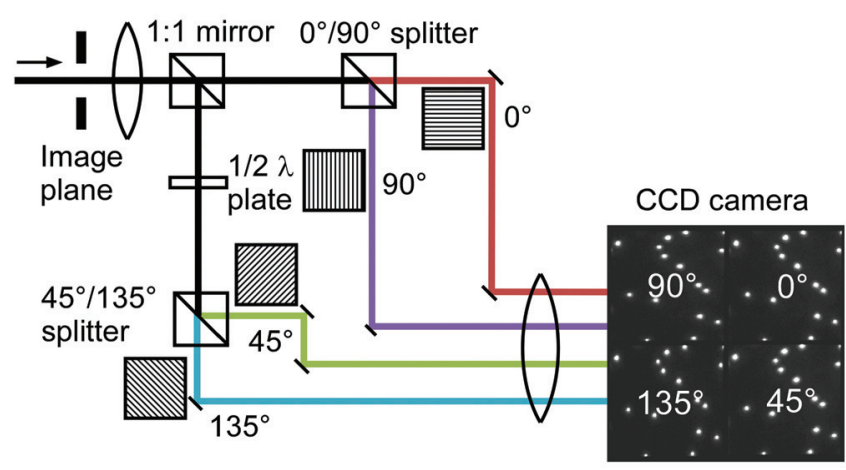

\section{B Lambda phage DNA stained with TOTO-1}

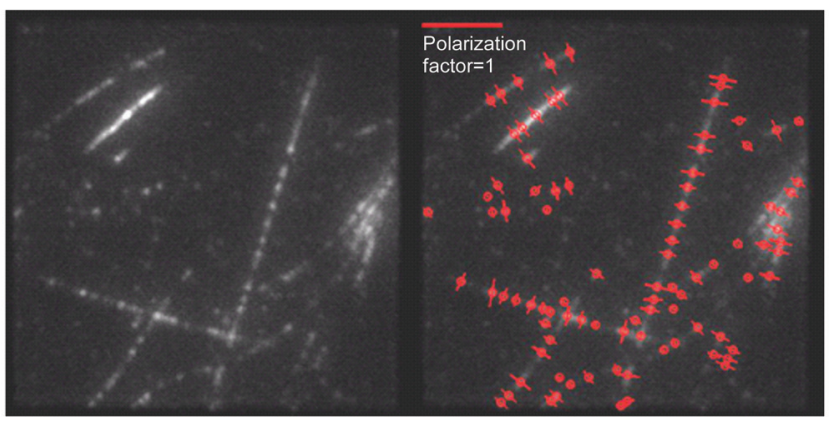

Figure 7. (A) Schematic diagram of the instantaneous fluorescence polarizing microscope ("FluoPolScope"). (B) Orientation analysis of TOTO1 fluorophores intercalated into stretched lambda phage DNA bound to a glass coverslip. Both left and right panels show the TOTO1 fluorescence image. The right panel includes an overlay of red lines that indicate the orientation and degree of polarized fluorescence. The length of the lines represents the polarization factor (P-factor), which varies between 0 for unpolarized fluorescence and 1 for fully polarized fluorescence.

birefringence, comes with many directional dynamics of life phenomena from cell motility, including muscle contraction, and even signaling in the nervous system. Larry Cohen first observed birefringence changes during nerve activity (Cohen et al., 1968, 1970), a phenomenon that is now being revisited in the context of whole brain slices (Koike-Tani et al., 2015). We are also combining light-field imaging with comprehensive polarization analysis to determine more fully the 3D polarization parameters in every resolved specimen volume (Oldenbourg, 2008).

This review recounts but a thin slice of Shinya Inoué's legacy, from a personal perspective of his MBL colleagues, who had the great fortune and pleasure to work with his guidance and inspiration for developing new advances in polarized light microscopy. Shinya Inoué's broader legacy for microscopy has contributed to a confluence of spectacular new optical techniques and a plethora of ways to highlight and label cell structures and functions. These have 
set a new stage upon which light microscopy advances our understanding of living cells and life itself.

\section{Acknowledgment}

The authors are immensely grateful to Shinya Inoué, who was a demanding, yet patient, and exacting, yet generous mentor; who taught by example. Preparation of this manuscript was supported by grants from the National Institutes of Health (no. GM100160 to TT; no. GM101701 to MS; and no. GM114274 to RO); and by the Marine Biological Laboratory start-up funds from the Inoué Family Endowment, to TT.

\section{Literature Cited}

Allen, R. D., N. S. Allen, and J. L. Travis. 1981. Video-enhanced contrast, differential interference contrast (AVEC-DIC) microscopy: a new method capable of analyzing microtubule-related motility in the reticulopodial network of Allogromia laticollaris. Cytoskeleton 1: 291302.

Axelrod, D. 1989. Fluorescence polarization microscopy. Methods Cell Biol. 30: 333-352.

Byrne, J. A., D. A. Pedersen, L. L. Clepper, M. Nelson, W. G. Sanger, S. Gokhale, D. P. Wolf, and S. M. Mitalipov. 2007. Producing primate embryonic stem cells by somatic cell nuclear transfer. Nature 450: 497-502.

Cohen, L. B., R. D. Keynes, and B. Hille. 1968. Light scattering and birefringence changes during nerve activity. Nature 218: 438-441.

Cohen, L. B., B. Hille, and R. D. Keynes. 1970. Changes in axon birefringence during the action potential. J. Physiol. 211: 495-515.

DeMay, B. S., X. Bai, L. Howard, P. Occhipinti, R. A. Meseroll, E. T. Spiliotis, R. Oldenbourg, and A. S. Gladfelter. 2011a. Septin filaments exhibit a dynamic, paired organization that is conserved from yeast to mammals. J. Cell Biol. 193: 1065-1081.

DeMay, B. S., N. Noda, A. S. Gladfelter, and R. Oldenbourg. 2011b. Rapid and quantitative imaging of excitation polarized fluorescence reveals ordered septin dynamics in live yeast. Biophys. J. 101: 985994.

Engelmann, T. W. 1875. Contractilität und Doppelbrechung. Pflïg. Arch. Eur. J. Physiol. 11: 432-464.

Hodgkin, A. L., A. F. Huxley, W. Feldberg, W. A. H. Rushton, R. A. Gregory, and R. A. McCance. 1977. The Pursuit of Nature: Informal Essays on the History of Physiology. Cambridge University Press, Cambridge, U.K.

Inoué, S. 1951. Studies of the Structure of the Mitotic Spindle in Living Cells with an Improved Polarization Microscope. Doctoral thesis. Princeton University, Princeton, NJ.

Inoué, S. 1952a. The effect of colchicine on the microscopic and submicroscopic structure of the mitotic spindle. Exp. Cell Res. Suppl. 2: 305-318.

Inoué, S. 1952b. Studies on depolarization of light at microscope lens surfaces: 1 . The origin of stray light by rotation at the lens surfaces. Exp. Cell Res. 3: 199-208.

Inoué, S. 1953. Polarization optical studies of the mitotic spindle. I. The demonstration of spindle fibers in living cells. Chromosoma 5: 487500 .

Inoué, S. 1964. Organization and function of the mitotic spindle. Pp. 549-598 in Primitive Motile Systems in Cell Biology, R. H. Allen and N. Kamiya, eds. Academic Press, New York.

Inoué, S. 1981a. Cell division and the mitotic spindle. J. Cell Biol. 91: 131s-147s.
Inoué, S. 1981b. Video image processing greatly enhances contrast, quality, and speed in polarization-based microscopy. J. Cell Biol. 89: 346-356.

Inoué, S. 1986. Video Microscopy. Plenum Press, New York.

Inoué, S. 2008a. C Collected Works of Shinya Inoué: Microscopes, Living Cells, and Dynamic Molecules. World Scientific, Singapore.

Inoué, S. 2008b. Microtubule dynamics in cell division: exploring living cells with polarized light microscopy. Annu. Rev. Cell Dev. Biol. 24: $1-28$.

Inoué, S., and W. L. Hyde. 1957. Studies on depolarization of light at microscope lens surfaces. II. The simultaneous realization of high resolution and high sensitivity with the polarizing microscope. J. Biophys. Biochem. Cytol. 3: 831-838.

Inoué, S., and E. D. Salmon. 1995. Force generation by microtubule assembly/disassembly in mitosis and related movements. Mol. Biol. Cell 6: 1619-1640.

Inoué, S., and H. Sato. 1966. Deoxyribonucleic acid arrangement in living sperm. Pp. 209-248 in Molecular Architecture in Cell Physiology, T. Hayashi and A. G. Szent-Gyorgyi, eds. Prentice Hall, Englewood Cliffs, NJ.

Inoué, S., and H. Sato. 1967. Cell motility by labile association of molecules. The nature of mitotic spindle fibers and their role in chromosome movement. J. Gen. Physiol. 50: 259-292.

Inoué, S., and L. G. Tilney. 1982. Acrosomal reaction of thyone sperm. I. Changes in the sperm head visualized by high resolution video microscopy. J. Cell Biol. 93: 812-819.

Inoué, S., O. Shimomura, M. Goda, M. Shribak, and P. T. Tran. 2002. Fluorescence polarization of green fluorescence protein. Proc. Natl. Acad. Sci. USA 99: 4272-4277.

Katoh, K., K. Yamada, F. Oosawa, and R. Oldenbourg. 1996. Birefringence measurements of the actin bundle in the acrosomal processes of Limulus sperm. Biol. Bull. 191: 270-271. (Abstract).

Koike-Tani, M., T. Tani, S. B. Mehta, A. Verma, and R. Oldenbourg. 2015. Polarized light microscopy in reproductive and developmental biology. Mol. Reprod. Dev. 82: 548-562.

LaFountain, J. R., Jr., and R. Oldenbourg. 2004. Maloriented bivalents have metaphase positions at the spindle equator with more kinetochore microtubules to one pole than to the other. Mol. Biol. Cell 15: $5346-5355$.

LaFountain, J. R., Jr., and R. Oldenbourg. 2014. Kinetochore-driven outgrowth of microtubules is a central contributor to kinetochore fiber maturation in crane-fly spermatocytes. Mol. Biol. Cell 25: 1437-1445.

LaFountain, J. R., Jr., C. S. Cohan, and R. Oldenbourg. 2011. Functional states of kinetochores revealed by laser microsurgery and fluorescent speckle microscopy. Mol. Biol. Cell 22: 4801-4808.

Liu, L., R. Oldenbourg, J. R. Trimarchi, and D. L. Keefe. 2000. A reliable, noninvasive technique for spindle imaging and enucleation of mammalian oocytes. Nat. Biotechnol. 18: 223-225.

McQuilken, M., S. B. Mehta, A. Verma, G. Harris, R. Oldenbourg, and A. S. Gladfelter. 2015. Polarized fluorescence microscopy to study cytoskeleton assembly and organization in live cells. Curr. Protoc. Cell Biol. 67: 4.29.1-4.29.13.

Mehta, S. B., M. Shribak, and R. Oldenbourg. 2013. Polarized light imaging of birefringence and diattenuation at high resolution and high sensitivity. J. Optics 15: 094007.

Mehta, S. B., M. McQuilken, P. La Riviere, P. Occhipinti, A. Verma, R. Oldenbourg, A. S. Gladfelter, and T. Tani. 2016. Dissection of molecular assembly dynamics by tracking orientation and position of single molecules in live cells. Proc. Natl. Acad. Sci. USA (in press).

Oldenbourg, R. 1996. A new view on polarization microscopy. Nature 381: $811-812$

Oldenbourg, R. 2008. Polarized light field microscopy: an analytical 
method using a microlens array to simultaneously capture both conoscopic and orthoscopic views of birefringent objects. J. Microsc. 231: $419-432$.

Oldenbourg, R., and G. Mei. 1995. New polarized light microscope with precision universal compensator. J. Microsc. 180: 140-147.

Oldenbourg, R., and M. Shribak. 2010. Microscopes. Pp. 28.1-28.62 in Handbook of Optics, Vol. 1, Geometrical and Physical Optics, Polarized Light, Components and Instruments, $3^{\text {rd }}$ ed., M. Bass, C. M. DeCusatis, J. M. Enoch, V. Lakshminarayanan, G. Li, C. MacDonald, V. N. Mahajan, and E. Van Stryland, eds. McGraw-Hill, New York.

Oldenbourg, R., E. D. Salmon, and P. T. Tran. 1998. Birefringence of single and bundled microtubules. Biophys. J. 74: 645-654.

Olmsted, J. B., and G. G. Borisy. 1973. Microtubules. Annu. Rev. Biochem. 42: 507-540.

Mehta, S. B., M. McQuilken, P. La Riviere, P. Occhipinti, A. Verma, R. Oldenbourg, A. S. Gladfelter, and T. Tani. 2016. Dissection of molecular assembly dynamics by tracking orientation and position of single molecules in live cells. Proc. Natl. Acad. Sci. USA (in press).

Ruska, E. 1980. The early development of electron lenses and electron microscopy. Microsc. Acta Suppl. Suppl. 5: 1-140.

Salmon, E. D. 1975. Spindle microtubules: thermodynamics of in vivo assembly and role in chromosome movement. Ann. N. Y. Acad. Sci. 253: 383-406.

Sato, H., G. W. Ellis, and S. Inoué. 1975 Microtubular origin of mitotic spindle form birefringence. Demonstration of the applicability of Wiener's equation. J. Cell Biol. 67: 501-517.

Schmidt, W. J. 1924. Die Bausteine des Tierkörpers in polarisiertem Lichte. F. Cohen, Bonn.

Schmidt, W. J. 1937. Die Doppelbrechung von Karyoplasma, Zytoplasma und Metaplasma. Gebrüder Bornträger, Berlin.

Schmidt, W. J. 1939. Doppelbrechung der Kernspindel und Zugfasertheorie der Chromosomenbewegung. Z. Zellforsch. Mikrosk. Anat. Abt. B Chromosoma 1: 253-264.

Shribak, M. 2011. Complete polarization state generator with one variable retarder and its application for fast and sensitive measuring of two-dimensional birefringence distribution. J. Opt. Soc. Am. A. Opt. Image Sci. Vis. 28: 410-419.

Shribak, M. 2013. Quantitative orientation-independent differential interference contrast microscope with fast switching shear direction and bias modulation. J. Opt. Soc. Am. A. Opt. Image Sci. Vis. 30: $769-782$.

Shribak, M. 2015. Polychromatic polarization microscope: bringing colors to a colorless world. Sci. Rep. 5: 17340

Shribak, M., and S. Inoué. 2006. Orientation-independent differential interference contrast microscopy. Appl. Opt. 45: 460-469.

Shribak, M., and R. Oldenbourg. 2003. Techniques for fast and sensitive measurements of two-dimensional birefringence distributions. Appl. Opt. 42: 3009-3017.

Shribak, M., S. Inoué, and R. Oldenbourg. 2002. Polarization aberrations caused by differential transmission and phase shift in highnumerical aperture lenses: theory, measurement, and rectification. Opt. Eng. 41: 943-954.

Shribak, M., J. R. LaFountain, Jr., D. Biggs, and S. Inoué. 2008. Orientation-independent differential interference contrast microscopy and its combination with an orientation-independent polarization system. J. Biomed. Opt. 13: 014011.

Tilney, L. G., and S. Inoué. 1982. Acrosomal reaction of Thyone sperm. II. The kinetics and possible mechanism of acrosomal process elongation. J. Cell Biol. 93: 820-827.

Valentin, G. G. 1861. Die Untersuchung der Pflanzen- und der Thiergewebe in polarisiertem Lichte. Verlag von Wilhelm Engelmann, Leipzig.

Vrabioiu, A. M., and T. J. Mitchison. 2006. Structural insights into yeast septin organization from polarized fluorescence microscopy. $\mathrm{Na}$ ture 443: 466-469.

Vrabioiu, A. M., and T. J. Mitchison. 2007. Symmetry of septin hourglass and ring structures. J. Mol. Biol. 372: 37-49. 